\title{
Determination of Ion Frequencies in a Quadrupole Ion Trap By Using a Fast Direct Current Pulse as Pump and a Laser Probe
}

\author{
Stephen A. Lammert, ' Curtis D. Cleven, and R. Graham Cooks \\ Department of Chemistry, Purdue University, West Lafayette, Indiana, USA
}

\begin{abstract}
A new technique has been developed which allows the direct measurement of frequencies of ions trapped in a quadrupole ion trap mass spectrometer. This pump/probe method employs a fast direct current (DC) pulse (pump) to displace a kinetically cooled ion population from the center of the trap, and a laser (probe) which recognizes when ions reappear at the center of the trap by the formation of photodissociation fragments. The translationally excited ions undergo periodic motion within the confines of the ion trap, and this periodic motion can be followed by recording the intensity of the photodissociation fragment as a function of the delay time between the DC pump and the laser probe. The DC pulse has a rise time of $15 \mathrm{~ns}$; data are taken $1 \mathrm{~ms}$ after its application to allow stable ion motion to be sampled. Sampling of the ion cloud is done at 50 ns intervals, and fast Fourier transformation of the time-based data yields the ion frequencies and their relative magnitudes. Data are reported for ions derived from acetophenone $(\mathrm{m} / \mathrm{z} 105)$ and 1,4-cyclohexadiene $(m / z 80)$ under various trapping conditions corresponding to different Mathieu $\mathrm{q}_{\mathrm{z}}$ values. The measured fundamental secular frequencies, $\mathrm{f}_{\mathrm{z}}$ and $\mathrm{f}_{\mathrm{r}}$, are found to agree well with those predicted. The presence of higher order multipole contributions to the trapping field is evident from such ion frequencies as the drive frequency, $\mathrm{f}_{\mathbf{R F}}$. The ability to measure ion frequencies under operating conditions provides a new tool for comparing simulated and experimental data. Simulation data from the program ITSIM, modified to account for the effects of collisions, are shown to predict the major frequency components observed in the experimental data. (J Am Soc Mass Spectrom 1994, 5, 29-36)
\end{abstract}

A fundamental property of an ion stored in a quadrupole ion trap [1] is the set of frequencies associated with its periodic motion. Separate sets of frequencies characterize motion in the radial (r) and axial ( $\mathrm{z}$ ) directions. In both cases, the lowest and typically most important component of these sets is termed the fundamental secular frequency. In real, as opposed to ideal, traps, contributions from dipole, hexapole, and higher order fields, whether adventitious or deliberately applied, introduce additional component frequencies. The relative magnitudes of all the individual frequencies determine the ion trajectory; for particular operating parameters, they determine whether ions can be trapped in the device. In addition to their significance in describing ion behavior, knowledge of ion frequencies is required to manipulate trapped ions by techniques such as resonance excitation [2], mass range extension [3], and radiofrequency (RF)-only isolation [4].

The angular frequencies $\left(\omega_{n, u}\right)$ imposed on the ion

\footnotetext{
Present address: Oak Ridge National Laboratory, P.O. Box 2008, M/S 6120, Oak Ridge, TN 37831-6120.

Address reprint requests to $R$. Graham Cooks, Department of Chemistry, Purdue University, West Lafayette, IN 47907.
}

by a pure quadrupole electric field are described [1] in terms of the parameter, $\boldsymbol{\beta}_{\mathrm{u}^{\prime}}$ and are given as

$$
\begin{gathered}
\omega_{\mathrm{n}, \mathrm{u}}=\left(\mathrm{r}+\frac{\beta_{\mathrm{u}}}{2}\right) \Omega \quad 0 \leq n<\infty \\
\omega_{\mathrm{n}, \mathrm{u}}=-\left(\mathrm{n}+\frac{\boldsymbol{\beta}_{\mathrm{u}}}{2}\right) \Omega \quad-\infty<n<0
\end{gathered}
$$

where $\Omega$ is the RF drive angular frequency, and $\mathbf{n}$ is an integer $(n= \pm 0,1,2, \ldots)$. The corresponding ion frequencies are designated $f_{n, u}$, the fundamental secular frequency being designated as $\mathbf{f}_{\mathbf{z}}$. The parameter, $\beta_{u}$, which describes ion motion is a complicated function of the Mathieu parameters, $a_{u}$ and $q_{u}$. However, ion motion is simplest at low $q_{u}$ values, where $u$ is $r$ for the radial direction or $\mathrm{z}$ for the axial direction [5]; at $\mathfrak{q}_{\mathfrak{u}}$ values less than 0.4 , it is described with reasonable accuracy in terms of the simple harmonic oscillator (pseudopotential) model of Dehmelt. In this model the ion oscillates at its axial and radial fundamental secular frequencies in separate potential wells, and at these low values of $q_{u}$ the parameter $\beta_{u}$ is approxi- 
mately given by

$$
\beta_{\mathrm{u}}^{2}=\mathrm{a}_{\mathrm{u}}+\mathrm{q}_{\mathrm{u}}^{2} / 2
$$

In the present study, more accurate methods of calculating $\beta_{\mathrm{u}}$ are used [1].

Work has been done with the goal of measuring ion frequencies under various conditions. In many ion trap experiments, ions are detected on the basis of resonance absorption and this provides a means of measuring frequencies. André and co-workers [6] were able to determine the fundamental secular frequency of an ion by observing intensity deviations in the time-of-flight signal of ejected ions. Vedel $[7,8]$ measured ejection signals using quadrupolar excitation to determine ion frequencies. Others have attempted to measure frequencies in Penning traps [9, 10]. Recent experiments by Yost and co-workers [11] and Todd et al. [12] have used resonance ejection from the ion trap to characterize ion frequencies. This method is very useful but is limited by the fact that ions begin to resonate with an applied field at frequencies which are near but not at the desired frequency [13]. Most important, none of the above experiments measure frequencies of motion of stable trapped ions.

An alternative approach to characterizing ion motion is based on ion tomography [14]. In these experiments, a UV-laser, which could be translated across the trap in the axial direction, was used to photodissociate the benzoyl cation. These experiments monitored the time-averaged ion distribution under a variety of operating conditions. In more recent tomography work, the trigger for the laser was phase-locked to an alternating current signal used to provide a dipole field for resonance excitation of the trapped ion population [15]. By sampling the ion cloud at the same point in its periodic motion, it was also possible to measure the axial component of the fundamental secular frequency, $f_{z}$

This article describes an alternative method to measure ion frequencies in the ion trap. This "pump/probe" method uses a fast DC pulse to electrostatically displace the ions and to cause them to oscillate within the ion trap. A laser probe is used to recognize the reappearance of ions at the center of the trap by generating and then measuring the intensity of a photodissociation product ion. The operating conditions are such that the photodissociation fragments are trapped, allowing them then to be ejected and detected. The measurement is made as a function of delay time between the pump and probe events. The DC pulse can be applied in either the axial or radial direction to displace the ions from the center of the trap. If the DC pulse is applied simultaneously to both endcaps, the ions are translated radially. Alternatively, the ions are translated axially if the DC pulse is applied to one endcap while the other is grounded. As will be seen later, neither procedure activates the ions exclusively in one direction.
A unique aspect of these experiments stems from the fact that use of a fast DC pulse not only rapidly moves the ion population away from the center of the trap but also forces the ions into phase coherence. When the ion position is subsequently probed with a laser, a time-varying photodissociation fragment signal results. This signal contains the fundamental secular frequency of the ion, often as the dominant component of ion motion. It also contains frequencies which correspond to higher order solutions to the Mathieu equation and frequencies associated with nonlinear fields. The measurement of these frequencies and their amplitudes is accomplished without changing the trap operating parameters, and unperturbed frequencies are therefore measured.

\section{Experimental}

All pump/probe experiments were performed using a prototype ion trap mass spectrometer (ITMS), which is described elsewhere [16]. Helium was used as the buffer gas for all experiments at an uncorrected pressure of $2 \times 10^{-5}$ torr as measured with a BayertAlpert-type ionization gauge. The experiments were performed with two compounds, acetophenone and 1,4-cyclohexadiene. Samples were introduced into the vacuum chamber via a Granville-Phillips leak valve at a partial pressure of $2 \times 10^{-6}$ torr. Ionization was by electron impact and the desired parent ion was iso lated by applying a DC potential and bringing it to the apex of the stability diagram using an appropriate combination of DC and RF potentials.

A home-built, high voltage DC pulser [17] was used to apply a fast, low amplitude DC pulse to one or both of the endcap electrodes to move the ions away from their kinetically cooled positions in the center of the ion trap. Axial translation of the ions was achieved by applying the DC pulse to a single endcap while the other endcap was grounded. Simultaneous application of the DC pulse to both endcaps was used for radial translation. A pulse amplitude of $10-50 \mathrm{~V}$ was used for axial translation and $100 \mathrm{~V}$ was used for radial translation. The pulse width for all experiments was 2 $\mu \mathrm{s}$, and it was always initiated when the RF was at zero phase angle.

A Lumonics Excimer-700 Series $\mathrm{XeCl}$ laser (Lumonics Inc., Ontario, Canada) was used to probe the ions after translational activation. The laser beam was introduced into the ion trap through the ring electrode by using the optical system shown in Figure 1. The beam, which was $1 \mathrm{~mm}$ in diameter, was steered through a $1.3 \mathrm{~mm}$ diameter aperture drilled in the center of the ring electrode. It was then passed through a matching exit aperture and was stopped by a $1 \%$ transmission filter mounted on a heat sink.

The quality of the experiment relies on the synchronization of the DC pulse and the laser. A custom-built circuit was used to provide a TTL pulse which was phase-locked to the $\mathrm{RF}$ at zero $\mathrm{RF}$ phase angle. This 


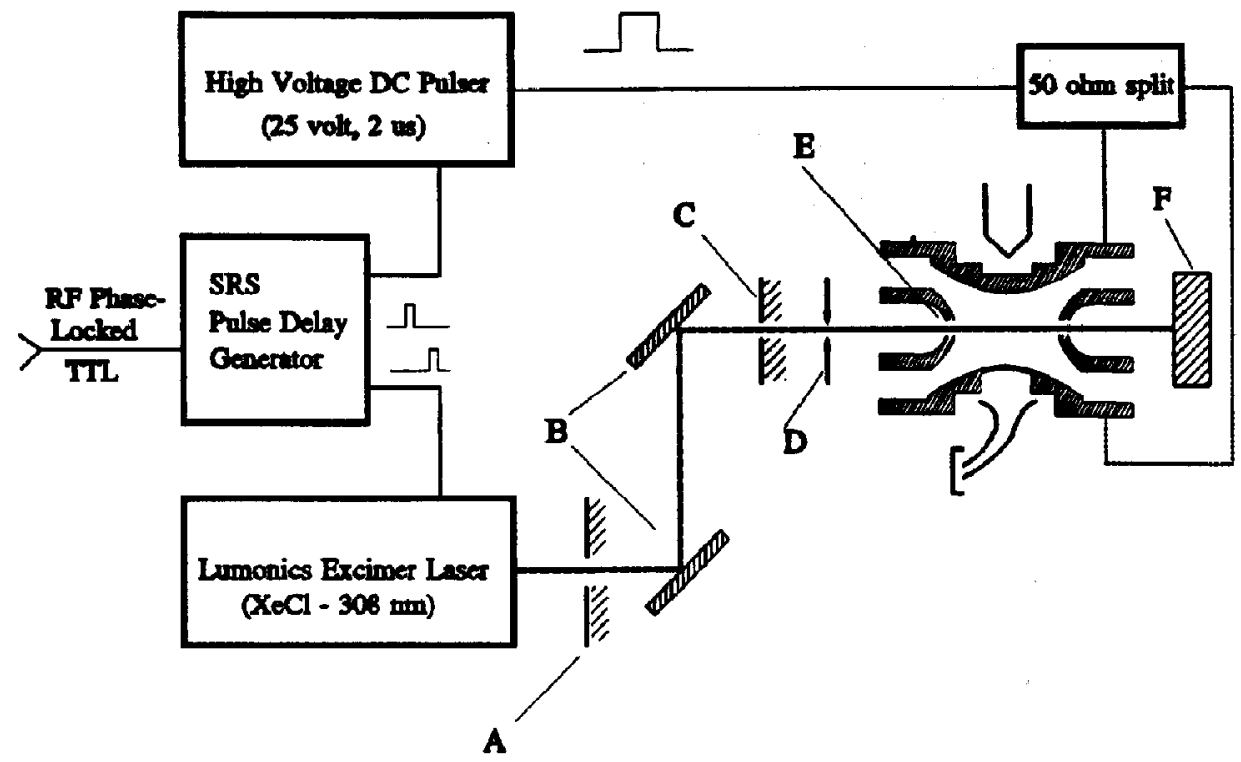

Figure 1. Schematic of the $\mathrm{DC}$ pulse pump/laser probe experiment. $\mathrm{A}=0.95 \mathrm{~cm}$ diameter aperture; $\mathrm{B}=$ excimer mirrors; $\mathrm{C}=0.19 \mathrm{~cm}$ diameter aperture; $\mathrm{D}=0.05-1.1 \mathrm{~cm}$ diameter adjustable iris; $\mathrm{E}=0.13 \mathrm{~cm}$ diameter ring electrode aperture; $\mathrm{F}=$ beam stop.

control pulse activated a Stanford Research Systems (Sunnyvale, CA) Model DG535 Pulse Delay Generator which precisely controlled the triggering of the $D C$ pulser and the laser. After RF/DC isolation of the selected parent ion, the RF level was lowered to the desired $\mathfrak{q}_{u}$ value while the $a_{u}$ value was set to zero. A delay of $1 \mathrm{~ms}$ allowed the ions to stabilize at this $\mathrm{q}_{z}$ value. The DC pulse was then applied for $2 \mu s$, starting at zero RF phase angle. Another $1 \mathrm{~ms}$ delay was employed prior to the firing of the laser to allow the ions to achieve stable periodic motion. The delay time was then increased from $1 \mathrm{~ms}$ in $50 \mathrm{~ns}$ increments and the experiment was repeated until a sufficient number of time increments had been sampled. For all experiments, the photofragments had $q_{u}$ values which allowed them to fall within the stability region so that they could be trapped for subsequent analysis. These ions were ejected and detected using the normal mass-selective instability scan [18]. The photodissociation fragment intensity (in arbitrary peak area units) was recorded at each time step. In addition, the intensity was recorded every 500 ns with the DC pulser turned off to provide a baseline. The ion frequencies and their relative magnitudes were obtained by fast Fourier transformation (FFT) of the time-based data.

\section{Results and Discussion}

\section{Spatially and Temporally Resolved Photodissociation}

The pump/probe technique employs a fast DC pulse to displace a kinetically cooled ion population away from the center of the ion trap. The ions are moved coherently by electrostatic repulsion due to the DC pulse; the short duration and relatively low amplitude of the DC pulse excludes the alternative possibility that resonance excitation due to appropriate frequency components in the pulse is responsible for ion activation. A laser probe is used to perform photodissociation and so to recognize when ions reappear at the center of the trap. Phatodissociation was used previously by Hemberger et al. [14] in ion tomography studies to monitor the spatial distribution of ions in the trap under various operating conditions. The advantage of photodissociation is that it provides a means by which the motion of trapped ions can be studied without perturbing the operating conditions of the system.

It is desirable that the sample be chosen so that photodissociation of a mass-selected ion results in a single product with high efficiency, For this reason, the benzoyl cation $\left(\mathrm{C}_{6} \mathrm{H}_{5} \mathrm{CO}^{+}\right), m / z \quad 105$, which results from electron ionization of acetophenone followed by methyl radical loss, was isolated and selected as the parent ion. It displays a high absorptivity at the xenon chloride excimer wavelength at $308 \mathrm{~nm}$, and photodissociation at this wavelength yields the product ion, $m / z 77$.

I'o perform experiments at $\mathrm{q}_{\mathrm{z}}$ values greater than 0.67 , a sample other than acetophenone must be chosen. A maximum $\mathrm{q}_{\mathrm{z}}$ value of 0.67 is allowed for the parent ion at $m / z 105$ to allow the photodissociation fragment to have a $\mathrm{q}_{z}$ value less than 0.908 (i.e., to lie within the stability boundary). For the higher $\mathrm{q}_{\mathrm{z}}$ data, 1,4 -cyclohexadiene was chosen as the sample. The molecular ion, $m / z 80, \mathrm{C}_{6} \mathrm{H}_{8}^{+*}$, was isolated and, in this case, photodissociation at $308 \mathrm{~nm}$ yielded $m / z 79$, $\mathrm{C}_{6} \mathrm{H}_{7}^{+}$, as the predominant product ion. It was more 
difficult to stabilize the pressure of 1,4-cyclohexadiene than that of acetophenone and it decreased slowly over the 2 to 3 hours required to perform the experiment. The combination of this factor and a lower absorptivity required that baseline be subtracted to normalize the data. Therefore, for the 1,4-cyclohexadiene studies, the "DC pulse off" fragment ion intensity was measured every $500 \mathrm{~ns}$ and was used to normalize the " $\mathrm{DC}$ pulse on" data.

After isolation of the parent ions, a $1 \mathrm{~ms}$ cooling period was used to cool the ions to the center of the ion trap. The ions remained in the center of the trap until they were translated either axially or radially by the application of a $2 \mu \mathrm{s}$, low voltage DC pulse. Prior to the application of the pulse, the ions possessed trajectories that were within the confines of the $1 \mathrm{~mm}$ diameter laser beam which was passed through the center of the ring electrode at $z=0$. If the $D C$ pulse was not applied, the intensity of the photodissociation fragment $m / z 77$ was constant over time, except for the long-term decrease associated with sample depletion.

\section{Axial Activation}

Figure $2 a$ shows the pump/probe results in the time domain for an experiment performed at $\mathrm{q}_{\mathrm{z}}=0.3$. For this experiment, the $\mathrm{DC}$ pulse, which had an amplitude of $10 \mathrm{~V}$, was applied to one endcap electrode to produce axial activation. The shortest delay time selected between the DC pulse and the laser was $1 \mathrm{~ms}$. This delay time was increased in steps of 50 ns to a maximum delay time of $1.028 \mathrm{~ms}$. The delay time shown on the $x$-axis of Figure 2 refers to the delay time between the DC pulse and the firing of the laser after waiting $1 \mathrm{~ms}$ for ion cooling. These experiments generally take 2 to 3 hours to perform and the delay time is adjusted manually.

Periodically, the maximum photodissociation signal for the data obtained with the pulse on attains the same signal intensity level as that obtained with the pulse off. This shows that the ion trajectories do not exceed the $1 \mathrm{~mm}$ diameter laser beam when the DC pulse is off. Hence the intensity of the $m / z 77$ fragment with the DC pulse off represents the maximum photodissociation intensity. This is consistent with results from Hemberger et al. [14] who determined that the kinetically cooled ion population is contained within $1 \mathrm{~mm}$ of the center of the trap under similar trapping conditions.

The large peaks seen in the time-domain spectrum shown in Figure $2 \mathrm{a}$ are apparently due to the axial secular frequency of the ions. To measure this frequency and those associated with the other peaks, FFT of the time-domain data was performed, and the result is shown in Figure $2 b$. Frequencies are assigned on Figure $2 \mathrm{~b}$ and also in Table 1 which includes results from other experiments at various $\mathrm{q}_{\mathrm{z}}$ values. The most obvious consequence of the method of sampling is that
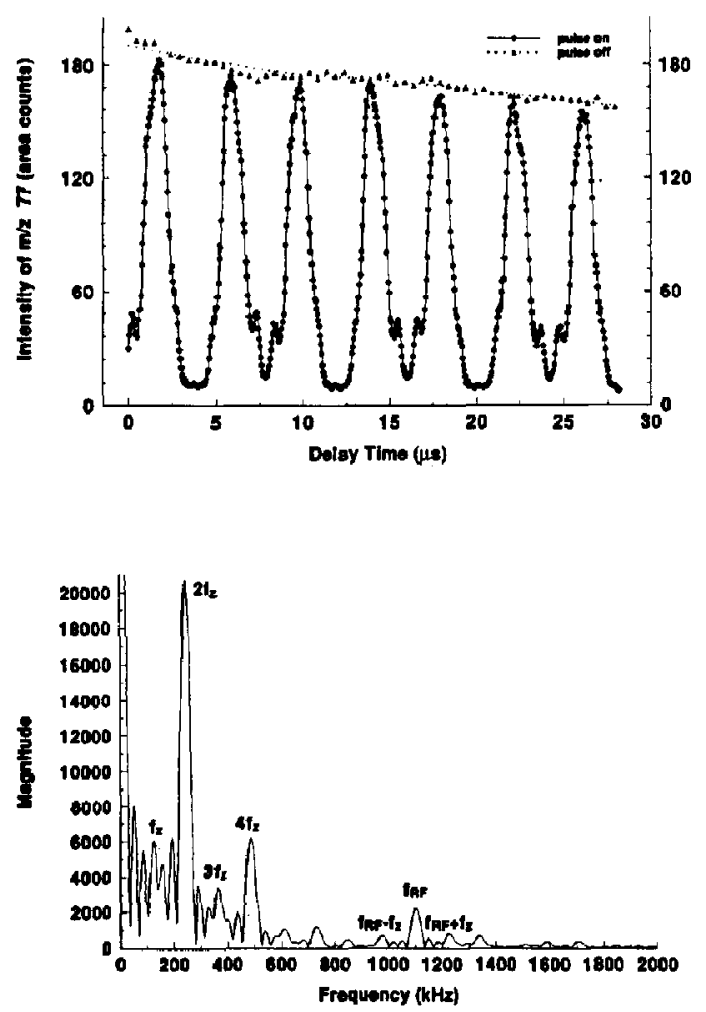

Figure 2. The time-domain (top) and frequency-domain (bottom) data for the $\mathrm{DC}$ pulse pump/laser probe experiment using a $10 \mathrm{~V}, 2 \mu \mathrm{s}$ axial DC pulse at a $\mathrm{q}_{z}$ value of 0.3 . The trapped ion is the benzoyl cation, $m / z$ 105, and the photofragment is $m / z 77$. The time delay refers to the length of time between the DC pulse and the firing of the laser after allowing a 1 ms cooling time.

an ion of a particular frequency could cross through the sampled volume in the center of the ion trap twice during one complete cycle and when this happens it would give rise to a measured frequency which is twice its actual frequency. The complex Lissajous motion of trapped inns is expected to allow some ions in an ion package to be sampled once and others twice per cycle.

In Figure 2b, the frequenry component with the largest magnitude is labeled $2 f_{z}$. It is quite probable that this peak results from the axial fundamental secular frequency, $f_{z}$, being sampled twice in one cycle (This was confirmed by moving the laser off center and observing an increase in $f_{z}$.) The measured frequency of $244 \mathrm{kHz}$ for $2 \mathrm{f}_{\mathrm{z}}$ agrees with the predicted frequency from eq 1 of $238 \mathrm{kHz}$ within the estimated uncertainty of $\pm 5 \%$ in the frequency measurement of the more abundant components. Also present in the frequency spectrum are the axial harmonics $3 f_{z}$ and $4 f_{z}$. The harmonic frequencies may be sampling or FFT artifacts or result from the nonlinear fields present in the nonideal ion trap [19]; although it is likely that the $4 f_{z}$ peak results both from sampling the second harmonic twice per cycle as well as from the fourth 
Table 1. Measured and calculated frequencies ${ }^{a}$ for axial activation at various $\mathrm{q}_{z}$ values

\begin{tabular}{|c|c|c|c|c|c|c|c|c|c|c|c|c|c|c|}
\hline & \multicolumn{2}{|c|}{0.3} & \multicolumn{2}{|c|}{0.5} & \multicolumn{2}{|c|}{0.6} & \multicolumn{2}{|c|}{$\begin{array}{c}q_{z}-V_{\text {alue }} \\
0.64\end{array}$} & \multicolumn{2}{|c|}{0.79} & \multicolumn{2}{|c|}{0.82} & \multicolumn{2}{|c|}{0.865} \\
\hline & Meas. & Calc." & Meas. & Calc. & Meas. & Calc. & Meas. & Calc. & Meas. & Calc. & Meas. & Calc. & Meas. & Calc. \\
\hline $\begin{array}{l}f_{z}^{c} \\
2 f_{z} \\
3 f_{z} \\
4 f_{z} \\
5 f_{z} \\
6 f_{z}\end{array}$ & $\begin{array}{l}124 \\
244 \\
366 \\
488\end{array}$ & $\begin{array}{l}119 \\
238 \\
356 \\
475 \\
594 \\
713\end{array}$ & $\begin{array}{r}195 \\
417 \\
615 \\
837 \\
1030\end{array}$ & $\begin{array}{c}206 \\
411 \\
617 \\
822 \\
1028 \\
1233\end{array}$ & $\begin{array}{r}242 \\
503 \\
747 \\
1011\end{array}$ & $\begin{array}{c}254 \\
508 \\
763 \\
1017 \\
1271 \\
1525\end{array}$ & $\begin{array}{r}246 \\
547 \\
808 \\
1098 \\
d \\
d\end{array}$ & $\begin{array}{c}275 \\
561 \\
826 \\
1101 \\
1377 \\
1652\end{array}$ & $\begin{array}{r}352 \\
732 \\
1101 \\
1479\end{array}$ & $\begin{array}{r}371 \\
742 \\
1112 \\
1483 \\
1854 \\
2225\end{array}$ & $\begin{array}{r}378 \\
791 \\
d \\
d \\
d\end{array}$ & $\begin{array}{c}396 \\
791 \\
1187 \\
1583 \\
1979 \\
2374\end{array}$ & $\begin{array}{r}454 \\
874 \\
1328 \\
1770\end{array}$ & $\begin{array}{r}443 \\
885 \\
1328 \\
1771 \\
2213 \\
2656\end{array}$ \\
\hline $\begin{array}{l}f_{R F} \\
2 f_{R F} \\
3 f_{R F}\end{array}$ & $\begin{array}{l}1101 \\
2201\end{array}$ & $\begin{array}{l}1100 \\
2200 \\
3300\end{array}$ & 1096 & $\begin{array}{l}1100 \\
2200 \\
3300\end{array}$ & $\begin{array}{l}1101 \\
2207\end{array}$ & $\begin{array}{l}1100 \\
2200 \\
3300\end{array}$ & $\begin{array}{l}1098 \\
2202\end{array}$ & $\begin{array}{l}1100 \\
2200 \\
3.300\end{array}$ & $\begin{array}{l}1101 \\
2202\end{array}$ & $\begin{array}{l}1100 \\
2200 \\
3300\end{array}$ & $\begin{array}{l}1098 \\
2204 \\
3305\end{array}$ & $\begin{array}{l}1100 \\
2200 \\
3300\end{array}$ & $\begin{array}{l}1101 \\
2202 \\
3308\end{array}$ & $\begin{array}{l}1100 \\
2200 \\
3300\end{array}$ \\
\hline $\begin{array}{l}f_{R F}+f_{z} \\
f_{R F}-f_{z} \\
2 f_{R F}+f_{z} \\
2 f_{R F}-f_{Z} \\
3 f_{R F}+f_{z} \\
3 f_{R F}-f_{Z}\end{array}$ & $\begin{array}{r}1225 \\
976 \\
\times\end{array}$ & $\begin{array}{c}1219 \\
981 \\
2319 \\
2081 \\
3419 \\
3181\end{array}$ & & $\begin{array}{c}1306 \\
894 \\
2406 \\
1994 \\
3506 \\
3094\end{array}$ & $\begin{array}{r}1345 \\
847\end{array}$ & $\begin{array}{r}1354 \\
846 \\
2454 \\
1946 \\
3554 \\
3046\end{array}$ & $\begin{array}{l}2458 \\
1921\end{array}$ & $\begin{array}{r}1375 \\
825 \\
2475 \\
1925 \\
3575 \\
3025\end{array}$ & $\begin{array}{r}1479 \\
732 \\
2570 \\
\end{array}$ & $\begin{array}{r}1471 \\
729 \\
2571 \\
1829 \\
3671 \\
2929\end{array}$ & $\begin{array}{r}1501 \\
698 \\
2592\end{array}$ & $\begin{array}{r}1496 \\
704 \\
2596 \\
7804 \\
3696 \\
2904\end{array}$ & $\begin{array}{r}1535 \\
649 \\
2644 \\
1770 \\
\\
2858\end{array}$ & $\begin{array}{r}1543 \\
657 \\
2643 \\
1757 \\
3743 \\
2857\end{array}$ \\
\hline $\begin{array}{l}f_{R F}+2 f_{z} \\
f_{R F}-2 f_{z} \\
f_{R F}+3 f_{z} \\
f_{R F}-3 f_{z}\end{array}$ & & $\begin{array}{r}1338 \\
862 \\
1456 \\
744\end{array}$ & $\begin{array}{r}1516 \\
708 \\
1709 \\
493\end{array}$ & $\begin{array}{r}1511 \\
689 \\
1717 \\
483\end{array}$ & $\begin{array}{r}1603 \\
607 \\
1862 \\
d\end{array}$ & $\begin{array}{r}1608 \\
592 \\
1863 \\
337\end{array}$ & $\begin{array}{c}d \\
547\end{array}$ & $\begin{array}{r}1651 \\
549 \\
1926 \\
274\end{array}$ & 352 & $\begin{array}{c}1842 \\
358 \\
2212 \\
12\end{array}$ & $\begin{array}{c}\text { d } \\
305\end{array}$ & $\begin{array}{r}7891 \\
309 \\
2287 \\
87\end{array}$ & $\begin{array}{c}d \\
215\end{array}$ & $\begin{array}{r}1985 \\
275 \\
2428 \\
228\end{array}$ \\
\hline $\begin{array}{l}2 f_{\mathrm{hF}}+2 \\
2 f_{\mathrm{RF}}-2 f_{2}\end{array}$ & & $\begin{array}{l}2438 \\
1962\end{array}$ & & $\begin{array}{l}2611 \\
1789\end{array}$ & & $\begin{array}{l}2708 \\
1692\end{array}$ & & $\begin{array}{l}2751 \\
1649\end{array}$ & & $\begin{array}{l}2944 \\
1458\end{array}$ & $\begin{array}{l}2988 \\
1408\end{array}$ & $\begin{array}{l}2991 \\
1409\end{array}$ & & $\begin{array}{l}3085 \\
1314\end{array}$ \\
\hline
\end{tabular}

All frequencies are in $\mathrm{kHz}$; $\mathrm{AF}$ drive frequency is $1.1 \mathrm{MHz}$.

b The predicted ion frequencies where $f_{z}$ is calculated from eq 1 are listed, in italics.

'The fundamental secular frequency is denoted $f_{z}$. The harmonics $\left(n f_{z}\right)$, the main $R F$ drive frequency $\left(f_{R F}\right)$, and various combination frequencies are also indicated.

Detected, but not messurable.

harmonic. The frequency of $1.1 \mathrm{MHz}$ corresponding to the $R F$ drive frequency, $f_{\mathrm{KF}}$, is also seen as are sidebands of the RF drive frequency which are labeled $f_{R F}-f_{x}$ and $f_{R F}+f_{z}$. The RF drive frequency is assigned to the hexapole field contribution [19] while the sideband frequencies are due to the quadrupole and can be predicted from eqs 1 and 2 for the cases where $\mathbf{n}= \pm 1$.

As the value of $q_{u}$ is increased, the pseudopotential well deepens and it is less easy to displace ions out near the electrodes where the nonlinear field components are strongest. As a result, the DC pulse amplitude must be increased to see the frequencies associated with the hexapole and octopole fields. These frequencies are described by Franzen [19b] as $\nu f_{R F} \pm n_{z} f_{z}$ where $\nu$ is an integer and $\mathrm{n}_{\mathrm{z}}$ is odd for the octopole component and odd or even for the hexapole component.

The effects of raising the $q_{x}$ value and the pulse amplitude can be seen in Figure 3 where a $25 \mathrm{~V}$ pulse is applied axially at a $q_{z}$ value of 0.6 . The time-domain spectrum shows that the ion motion is more complex. (It should be noted that the maximum signal intensities with the pulse on exceed those obtained with the pulse off. This occurs when the laser is not intersecting the z-axis perpendicularly so that the laser is sampling a cross section slightly away from the trap center.) In Figure $3 \mathrm{~b}$, the measured frequency, $2 \mathrm{f}_{\mathrm{z}}$ of $503 \mathrm{kHz}$ agrees well with the value of $508 \mathrm{kHz}$ predicted from eq 1. The RF drive frequency, $\mathrm{f}_{\mathbf{R F}}$, is present as are the $f_{\mathrm{KF}}+f_{\mathrm{z}}$ and $\mathrm{f}_{\mathrm{KF}}-\mathrm{f}_{\mathrm{z}}$ sideband frequencies. The frequencies $f_{R F} \pm 2 f_{z}$ and $f_{R F}+3 f_{z}$ may result from nonlinear field components.

Somewhere between $\mathrm{q}_{\mathrm{z}}=0.79$ and $\mathrm{q}_{\mathrm{z}}=0.82$, the $\mathrm{RF}$ drive frequency associated with the hexapole electric field component becomes the largest component of the ion motion. This frequency, $\mathrm{f}_{\mathrm{RF}}$, is clearly the largest frequency component in Figure 4 . This experiment employs a $30 \mathrm{~V}$ pulse at a $\mathrm{q}_{z}$ value of 0.865 . Harmonic frequencies of the $R F$ drive frequency are present up to $3 \mathrm{f}_{\mathrm{RF}}$. At high $\mathrm{q}_{\mathrm{u}}$ values, the higher order sidebands corresponding to the quadrupole field $\left(\mathrm{nf}_{\mathrm{RF}}\right.$ $\pm f_{z}$ ) are also more evident. It is difficult to compare the relative frequencies from experiments performed at different $q_{z}$ values, however, because the conditions were optimized to measure the frequencies and this required different pulse amplitudes. It is clear though, that the "stretched geometry" [20] of the Finnigan ion trap introduces additional nonlinear field components among which the octopole component is calculated to be the most significant." However, these calculations do not consider distortions produced by making holes in the electrodes, and the experimental values given

\footnotetext{
*R.K. Julian, Jr., Ph.D. Thesis, Purdue University, May 1993, gives 1\%-2\% octopole, while I. Franzen, Presentation at CBMS Workshop, Bremen, Germany, July 26-30, 1993, gives 1.44\% octopole, and 0.63\% dodecapole. Both calculations give $<0.1 \%$ hexapole.
} 

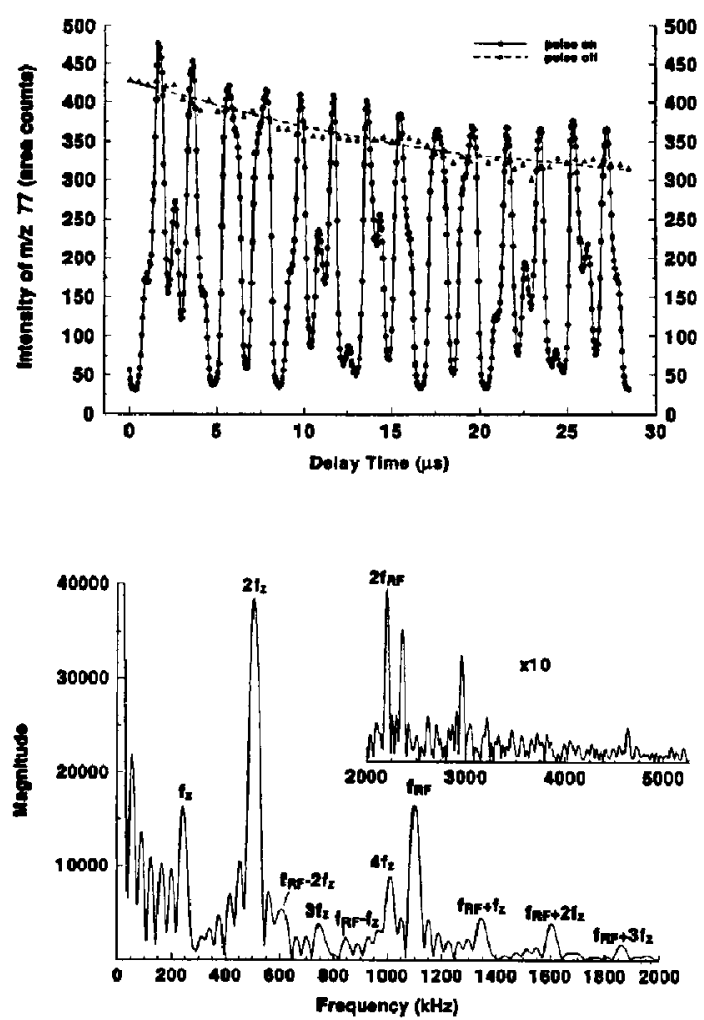

Figure 3. The time-domain (top) and frequency-domain (bottom) data for the $\mathrm{DC}$ pulse pump/laser probe experiment using a $25 \mathrm{~V}, 2 \mu \mathrm{s}$ axial DC pulse at a $\mathrm{q}_{z}$ value of 0.6 . The trapped ion is the benzoyl cation, $m / z$ 105, and the photofragment is $m / z 77$. The time delay refers to the length of time between the DC pulse and the firing of the laser after allowing a $1 \mathrm{~ms}$ cooling time.

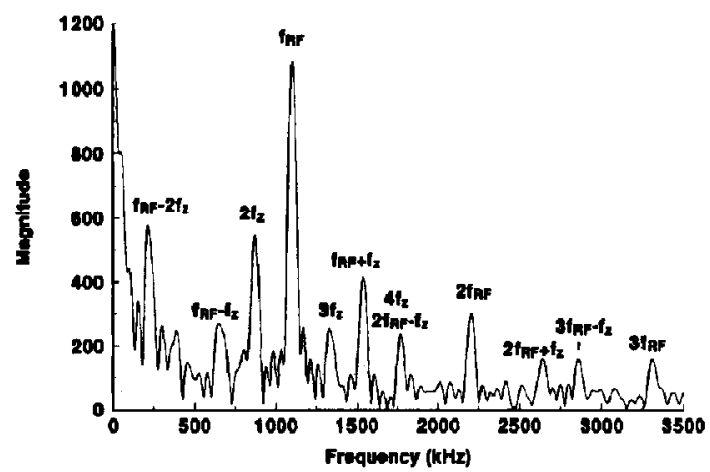

Figure 4. The frequency-domain data for the $D C$ pulse pump/laser probe experiment using a $50 \mathrm{~V}, 2 \mu \mathrm{s}$ axial DC pulse at a $\mathrm{q}_{z}$ value of 0.865 . The trapped ion is $\mathrm{m} / \mathrm{z} 80$ of 1,4-cyclohexadiene and the photofragment is $m / z$ 79. The time delay refers to the length of time between the $D C$ pulse and the firing of the laser after allowing a $1 \mathrm{~ms}$ cooling time. The peak at 1770 $\mathrm{kHz}$ probably results from contributions from both $4 \mathrm{f}_{z}$ and $2 f_{R F}-f_{z}$ and is labeled accordingly. here emphasize the importance of the resulting hexapole contributions.

\section{Radial Activation}

In an ideal quadrupole trap, axial and radial motion are uncoupled. However, even in an ideal quadrupole trap, the shapes of the electrodes are such that activation in only one direction is not possible. In the pump/probe experiment, the DC pulse will produce a small amount of activation in the radial motion accompanying the desired axial motion. Axial activation will also be accompanied by a small degree of radial activation due to the presence of the octopole fields. Hence, this effect was sought but not unambiguously found in the data. In Figure $3 \mathrm{~b}$, the peak at $242 \mathrm{kHz}$ could result from $2 f_{r}$, which has a predicted frequency of $238 \mathrm{kHz}$, as well as from $f_{z}$.

Radial activation can be achieved more successfully by simultaneously applying the DC pulse to both endcaps. A pulse voltage of about $100 \mathrm{~V}$ was used to displace the ion population from the center of the trap in the radial direction. Figure 5 illustrates both the time and frequency-domain data for the radial activation experiment at $\mathrm{q}_{\mathrm{z}}=0.5$. This corresponds to a $\mathrm{q}_{\mathrm{r}}$ value of 0.25 , which is low enough that simple inn motion is expected. Only the radial frequency, $2 \mathrm{f}_{\mathrm{r}}$, and the $\mathrm{nf}_{\mathrm{r}}$ harmonics are observed. No frequencies arising from axial motion are seen. This is not unexpected as the sensitivity to axial motion under the conditions of radial excitation should be small. In effect, the cylindrical laser beam is only intersecting a portion of the radial "disk." The overall sensitivity to both the radial and axial frequencies is reduced in this mode of activation and the frequency peaks are correspondingly broad.

\section{Simulation of Pump / Probe Experiment}

The pump/probe experiment may be simulated by using a set of programs based on ITSIM, a PC-based ion trap simulation program which integrates the equations of ion motion [21]. This program can be used to follow the position of the trapped ions in the $r-$ and $z$-directions as a function of time.

The ITSIM program uses a designated set of initial conditions to calculate the pusition of the ions as a function of time. The terminating positions and phase angles calculated by ITSIM are recorded to a file and may be used as the initial conditions for the next phase of the experiment. In this fashion, the program may be used to visualize the trajectories during the many different stages of the pump/probe experiment including the periods of ionization, kinetic energy cooling, DC pulse pumping, and laser probing. The ions are first generated in a program, ITIONIZE, a derivative of ITSIM that originates a set of ions with random RF phase angles [22]. The resulting positions and phase angles are used as the initial conditions for the cooling 

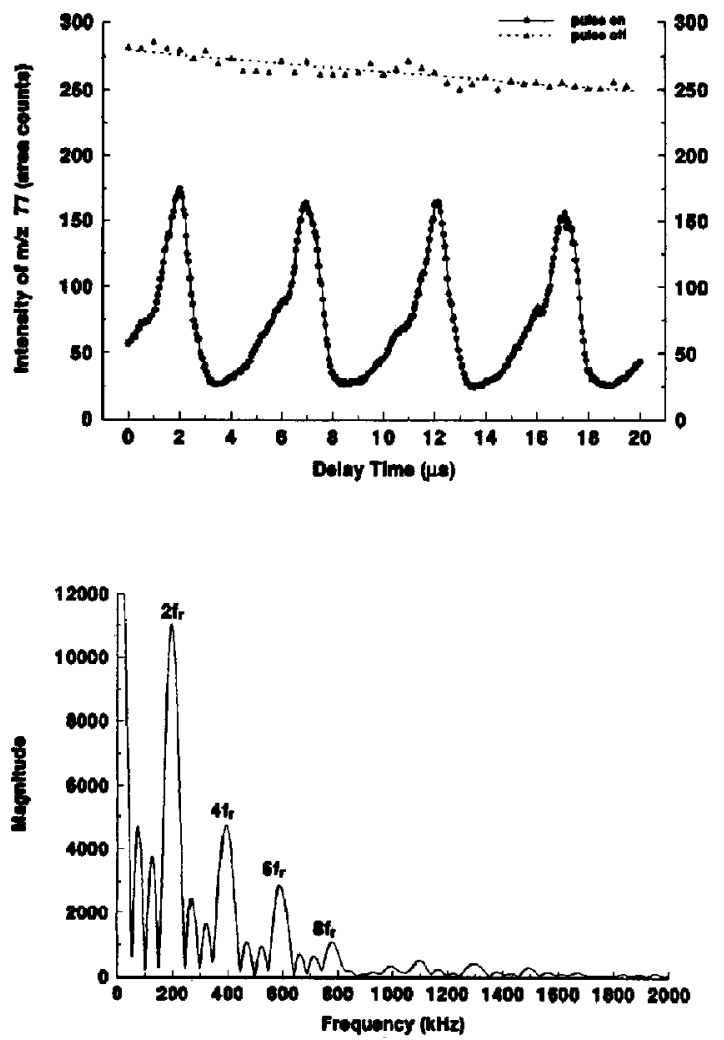

Figure 5. The time-domain (top) and frequency-domain (bottom) data for the $\mathrm{DC}$ pulse pump/laser probe experiment using a 100 volt, $2 \mu$ s radial $D C$ pulse at a $\mathrm{q}_{2}$ value of 0.5 . The trapped ion is the benzoyl cation, $m / z 105$, and the photofragment is $m / z$ 77. The time delay refers to the length of time between the DC pulse and the firing of the laser after allowing a $1 \mathrm{~ms}$ conling time.

stage of the experiment. The kinetically excited ions are cooled by implementing a dampening factor of $1 \times 10^{-3}$ representing the collisions with a buffer gas at a pressure of $2 \times 10^{-5}$ torr. In the third stage of the simulation, the DC pulse is applied and it is seen to effect phase coherence of the ions [22]. This is vital for the fourth stage where the presence of the ions at the center of the trap is probed by the laser beam.

Figure 6 illustrates the results of a simulation of the pump/probe experiment by using a $25 \mathrm{~V}$, axial DC pulse at a $\mathrm{q}_{\mathrm{z}}$ value of 0.6 . The simulation was performed for the fields associated with the actual "stretched" trap geometry rather than an idealized pure quadrupole geometry although endcap apertures were not considered. The phase coherence of the ions is evident in the plot of $\mathrm{z}$-position versus time. I'he photodissociation intensity represents the number of ions present within the cylindrical cross section of the laser beam which samples $1 \mathrm{~mm}$ in the $x$-and z-positions and all $y$-positions. The general pattern of this time-domain spectrum is similar to the experimental data shown in Figure 3a. The simulation yielded 490
$\mathrm{kHz}$ for $2 \mathrm{f}_{\mathrm{z}}$, which agrees with the experimentally measured frequency of $503 \mathrm{kHz} \pm 5 \%$. There is a set of shoulders in the simulation spectrum which correspond to motion with a frequency of $122 \mathrm{kHz}$. This may be due to radial motion which has a predicted frequency for $f_{r}$ of $119 \mathrm{kHz}$. However, the assignment is ambiguous because the predicted frequency of $f_{z} / 2$ is $127 \mathrm{kHz}$. A second simulation was performed at a $q_{z}$ value of 0.3 and gave a $2 f_{z}$ value of $238 \mathrm{kHz}$ while the pump/probe experiment yielded a frequency of 244 $\mathrm{kHz} \pm 5 \%$.

\section{Conclusion}

The component frequencies associated with the motion of trapped ions can be determined experimentally by using a new, DC pulse/laser probe technique. The success of this measurement stems from the fact that the DC pulse is able to move all ions rapidly away from the center of the ion trap and impart phase coherence to the ion population. This interpretation is confirmed by the results of simulations performed using the program ITSIM. The results showed an increasing contribution of the higher order sidebands $\left(n f_{R F} \pm f_{z}\right)$ with an increase in the $q_{u}$ value.

This technique holds promise for mapping the $\beta_{\mathrm{u}}$ $\mathrm{q}_{\mathrm{u}}$ relationship and as such should give complementary results to resonance ejection experiments of Eades and Yost [23]. The exact shape of the stability diagram for an actual, as opposed to an ideal, instrument is important in many applications and there is a special interest in the shape of the apex since this determines performance in RF/DC isolation experiments [24]. To perform such a mapping, many experiments, including those at non $\mathrm{a}_{\mathrm{z}}=0$ values, will need to be performed. The pump/probe experiment may also provide a means by which the frequencies associated with higher order field components may be studied. Exploration of the shifts in resonant ion frequencies which accompany common experimental conditions such as space charge, high buffer gas pressure, and highly abundant ions of similar mass, could also be undertaken by using this technique.

There is special interest in the results taken at $q_{z}=$ 0.64 and $\mathrm{q}_{\mathrm{z}}=0.79$ since these values are close to the octopole and hexapole nonlinear resonance values $\left(\beta_{z}=1 / 2\right.$ and $\beta_{z}=2 / 3$, respectively). Energy $a b-$ sorption from the external drive field might be expected to drive the ions further from the center and increase the magnitudes of those frequencies corresponding to nonlinear field components. This effect will be sought in future experiments in which the amplitude of the $\mathrm{DC}$ pulse is controlled.

Modifications to the experiment might lead to better data including improved frequency resolution. Photodissociation occurs at the center of the trap where the axial velocity of the ion is the greatest. Improved performance does result from off-center sampling. Another factor is that the laser beam is not focused and is 


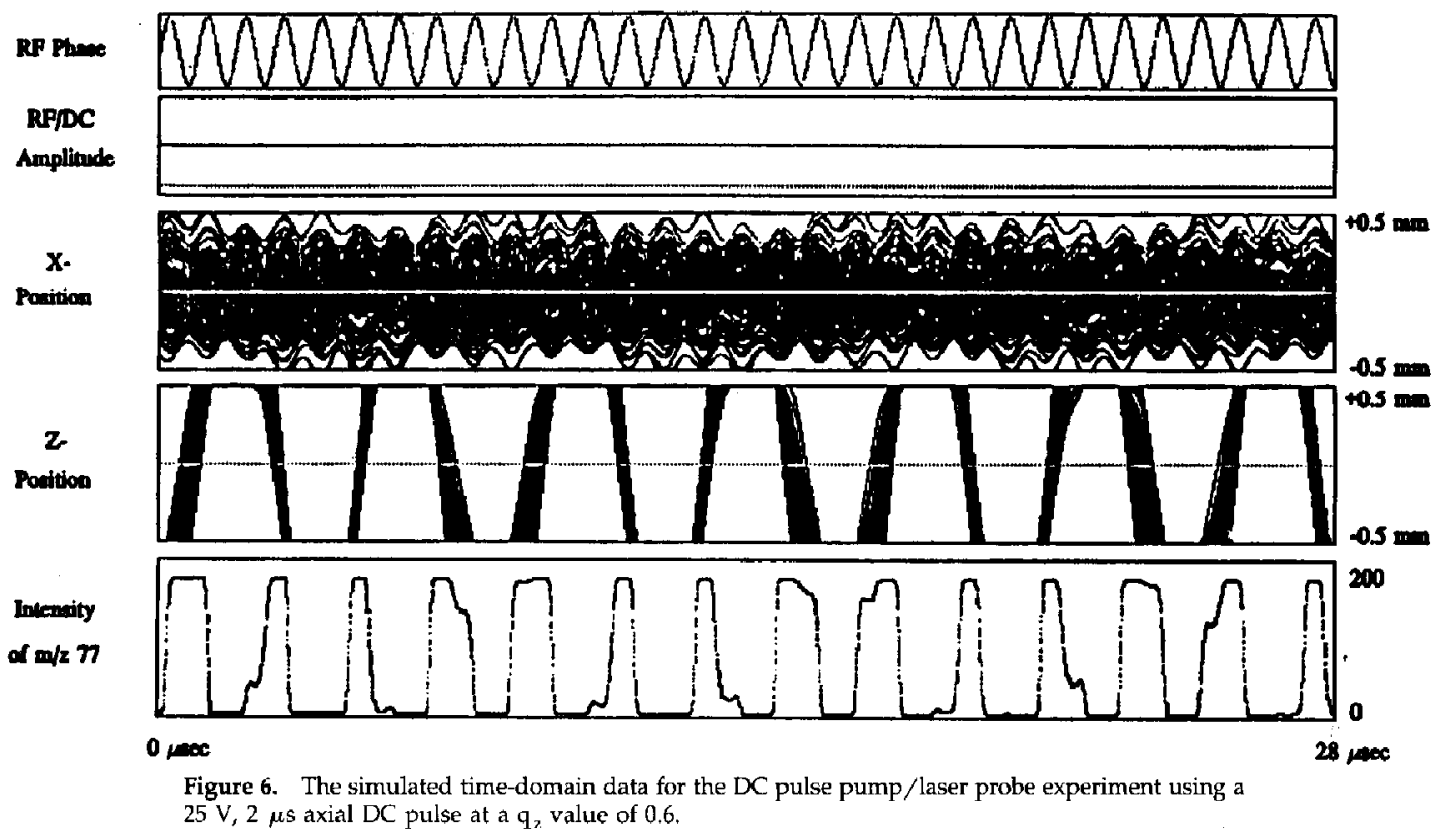

about $1 \mathrm{~mm}$ wide, and this results in the simultaneous sampling of a region of almost $10 \%$ of the axial dimension. Positioning a well-focused beam at the turning points of the axial motion, where the velocities are slowest, may provide higher quality frequency information. In addition, at these larger distances from the center of the trap, the strength of the nonlinear fields is increased and resulting nonlinear frequencies may be more easily detected there. The pump/probe experiment could be dramatically improved if a well-focused laser were to be translated along the axial axis. The combination of spatial resolution in laser tomography $[14,15]$ with the temporal resolution used here would provide a particularly powerful method of characterizing ion behavior in ion traps.

\section{Acknowledgments}

This work was supported by the National Science Foundation, CHE 87-21768 and 92-23791. The authors thank Randy Julian for assistance with the simulations.

\section{References}

1. March, R. E.; Hughes, R. J. Quadrupole Storage Mass Spectrometry: Wiley: New York, 1989.

2. Fulford, J. E.; Hoa, D.-N.; Hughes, R. J.; March, R. E.; Bonner, R. F.; Wong, G. J. J. Vac. Sci. Technol. 1980, 17, 829.

3. Kaiser, R. E., Jr.; Louris, J. N.; Amy, J. W.; Cooks, R. C. Rapld Commun. Mass Spectrom. 1989, 3, 225.

4. Kaiser, R. E., Jr.; Cooks, R. G.; Syka, J. E. P.; Stafford, G. C. Rapid Commun. Mass Spectrom. 1990, 4, 30.

5. Major, F. G.; Dehmelt, H. G. Phys. Rev. 1968, 170, 91.

6. Brincourt, G.; Catella, R.; Zerega, Y.; Andrér J. Chem. Phys. Lett. 1990, 174, 626.

7. Vedel, F. Int. J. Mass Spectrom. Ion Processes 1991, 108, R11.
8. Vedel, F. Int. I. Mass Spectrom: Ion Processes 1991, 106, 33.

9. Bate, D. J.; Dholakia, K.; Thompson, R. C.; Wilson, D. C. I Mod. Optics 1992, 39, 305.

10. Imajo, H.; Urabe, S.; Hayasaka, K.; Watanabe, M. J. Mod. Optics 1992, 39, 317.

11. Eades, D. M.; Johnson, J. V.; Yost, R. A. Proceedings of the 41st Annual ASMS Conference on Mass Spectrometry and Allied Topics; San Francisco, May 30-June 4, 1993; WP 163.

12. Todd, J. F. J.; Penman, A. D.; Thorner, D. A.; Smith, R. D. Rapid Commun. Mass Spectrom. 1990, 4, 108.

13. Morand, K. L., Ph.D. Thesis, Purdue University, December 1992.

14. Hemberger, P. H.; Nogar, N. S.; Williams, J. D.; Cooks, R. G.; Syka, J. E. P. Chem. Phys. Lett. 1992, 191, 405.

15. Williams, J. D.; Cooks, R. G.; Syka, J. E. P.; Hemberger, P. H.; Nogar, N. S. J. Am. Soc. Mass Spectrom. 1993, in press.

16. Louris, J. N.; Cooks, R. G.; Syka, J. E. P.; Kelley, P. E.; Stafford, G. C.; Todd, J. F. J. Anal. Chem. 1987, 59, 1677.

17. Lammert, S. A.; Cooks, R. G. J. Am. Soc. Mass Spectrom. 1991 , 2,487 .

18. Stafford, G. C.; Kelley, P. E.; Syka, J. E. F,; Reynolds, W. E.; Todd, J. F. J. Int. J. Mass Spectrom. Ion Processes 1984, 60, 85.

19. (a) Wang, Y.; Franzen, J. Int. I. Mass Spectrom. Ion Processes 1993, in press. (b) Compare Franzen, J., submitted, Wang, Y., Rapid Commun. Mass Spectrom., in press. (c) Franzen, J., Wang, Y., Int. J. Mass Spectrom. Ion. Processes 1991, 106, 63.

20. Louris, J. N.; Stafford, G. C., Jr.; Syka, J. E. P.; Taylor, D. Procedings of the 40th Annual ASMS Conference on Mass Spectrometry and Allied Topics; Washington DC, May 31-June 5, 1992; p 1003.

21. Reiser, H.-P.; Julian, R. K, Jr.; Cooks, R.G. Int. I. Mass Spectrom. Ion Processes 1992, 121, 49.

22. Julian, R. K., Jr.; Cooks, R. G. J. Am. Soc Mass Spectrom. submitted for publication.

23. Eades, D.M.; Y'ost, R.A. Rapid Commun. Mass Spectrom. 1992, 6,573 .

24. Louris, J. N.; Cooks, R, G; Syka, J. E. P.; Kelley, P. E. Stafford, G. C., Jr.; Todd, J. F. J. Anal. Chem. 1987, 59, 1677. 